\title{
LA EXPRESIÓN DE LA FUNCIÓN MANERA EN GRIEGO
}

This paper seeks to identify the marks for the semantic function Manner in Ancient Greek and to determine the conditions under which each of them is chosen. The following conclusions are reached: a grammatical function Manner exists in Greek which indicates that the lexeme with which some given morphemes combine denotes the way in which the predicate takes place. These marks are generally distributed according to the lexemes with which each of them can combine. The relationships of dependency can be denoted either by marking the function of the term or by its agreement with the governing element. This idea accounts for the remaining examples, in which different morphemes expressing Manner are found with the same lexeme.

1. Los manuales de sintaxis establecen que la función semántica Manera (término que utilizamos en lugar de "Modo", para evitar cualquier ambigüedad con la categoría del modo verbal) puede ser expresada en griego mediante procedimientos gramaticales distintos:

(1) a adverbios en - $\omega \varsigma,-\eta,-o v,-a$, etc. (cf. K.-G., II, p. 114),

b diferentes sintagmas preposicionales, como $a n \delta$ + Genitivo (cf. K.G., I, p. 459), 乌úv + Dativo (cf. K.-G., I, p. 467), $\varepsilon \xi$ + Genitivo (cf. K.-G., I, p. 461), $\varepsilon v+$ Dativo (cf. K.-G., I, p. 466), $\varepsilon l \varsigma$ + Acusativo (cf. K.-G., I, p. 471), dvá + Acusativo (cf. K.-G., I, p.

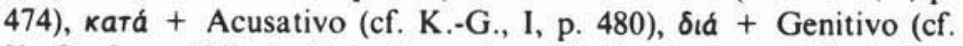
K.-G., I, p. 483), $\boldsymbol{\mu} \phi i+$ Acusativo (cf. K.-G., I, p. 491), $\varepsilon \pi i+$ Acusativo (cf. K.-G., I, p. 505), etc.

c oraciones subordinadas y elementos introducidos por $\dot{\omega} \varsigma, \tilde{\omega} \sigma \pi \varepsilon \rho$,

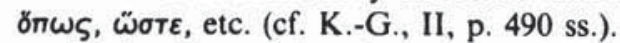

d participio concertado (cf. K.-G., II, pp. 77 s. y 86 s.; Rijksbaron 1984: p. 122 s.).

e participio absoluto (cf. K.-G., II, pp. 77 s. y 86 s.; Rijksbaron 1984: p. 122 s.).

En la medida en que yo sé, nunca se ha intentado determinar las condiciones que rigen la distribución de cada uno de los procedimientos de expresar la Manera, si es que realmente los procedimientos enumera- 
dos en (1) expresan la misma función semántica y, además, tienen el mismo significado. Esto es asi por dos causas, sobre todo: por un lado, la gramática griega ha atendido fundamentalmente a presentar la traducción que corresponde a los elementos gramaticales y no pone en relación formas distintas a las que se asigna la misma traducción o el mismo significado gramatical; por otro lado, en la gramática griega se suele adoptar un punto de vista exclusivamente morfológico, con el resultado de que elementos a los que se atribuye un mismo significado no son puestos en relación. No obstante, para hacer una descripción completa de la gramática, sería necesario precisar qué condiciones regulan la distribución de las formas gramaticales a las que se atribuye la expresión de una misma función y un mismo significado. Un objetivo, pues, de este artículo consiste en describir las reglas que rigen la distribución de los procedimientos que sirven para expresar la función semántica Manera.

Sin embargo, antes de abordar el objetivo ya expuesto, es preciso comprobar, con ayuda de criterios ajenos a la pura traducción, qué formas son susceptibles de expresar la función Manera. Éste es el otro objetivo de estas páginas, que necesariamente debemos abordar antes. Sólo cuando hayamos podido determinar qué formas son susceptibles de expresar Manera, estaremos en condiciones de abordar el problema de la distribución.

El contenido de este artículo está organizado del siguiente modo: en $\S 2$ expondremos y justificaremos brevemente el método que vamos a utilizar para intentar determinar los procedimientos susceptibles de expresar la función Manera. En $\S 3$ presentamos los datos pertinentes que muestren qué marcas formales expresan esta función. En $\S 4$ intentaré hacer ver que la distribución de los procedimientos para expresar la función Manera depende en gran medida de las posibilidades que cada morfema tiene de combinarse con determinados tipos de lexemas. Así, unos procedimientos de expresar Manera sólo se asocian con un tipo de lexemas, y otros con otro. En $\S 5$ presentaré una interpretación que explica la existencia de distintos procedimientos para expresar la Manera con los mismos lexemas. Ello nos permitirá discutir brevemente las diferencias existentes entre rección y concordancia gramatical. Finalmente, en $\S 6$ resumiré las conclusiones obtenidas '.

' La descripción gramatical de una función semántica debe incluir también otros datos (cf. 3.9) que aqui no hemos abordado por las razones expuestas en 3.9, y que si describe para el latin Vester (1983). Los datos que hemos analizado son los siguientes: hemos hecho una revisión sistemática de Tucidides, Demóstenes, I-III, VIII y XVIII; Platón, Apologia; y Homero. Además, hemos utilizado los ejemplos de K.- 
2. Los criterios que vamos a utilizar para intentar determinar qué procedimientos sirven para expresar la función semántica Manera son los tres siguientes:

a) El tipo de respuestas a interrogaciones parciales. Las interrogativas parciales preguntan por la identidad de un elemento del que el interrogativo parcial expresa la función. El interlocutor, siempre que haya condiciones normales de inteligibilidad y exista propósito de informar a quien hace la pregunta, responde con términos que desempeñan la misma función semántica que el interrogativo parcial que introduce la pregunta (cf. Pinkster 1972: p. 103 ss.; Rijksbaron 1976: pp. 9 ss., 33 ss.; de la Villa 1987).

b) La coordinación entre elementos morfológicos distintos, cuando al menos de uno de ellos se sabe, en virtud de otros criterios, que expresa la función Manera. La aplicación de este criterio se basa en el siguiente principio: la coordinación entre términos sólo es posible cuando estos términos están en el mismo nivel de la jerarquía sintáctica y, además, expresan la misma función (cf. Dik 1968; 1980). La coordinación está marcada en general por la presencia de una conjunción de coordinación, aunque también puede haber entre los términos coordinados mera aposición (coordinación mediante $\phi$ ). Sin embargo, la aposición y la correlación, en cuanto que no presentan marca formal en la lengua escrita cuando las formas morfológicas en aposición o correlación son distintas, pueden ser confundidas con la yuxtaposición [cf. infra c)]. Por ello, ni la aposición ni la correlación serán utilizadas como criterios para determinar qué formas son susceptibles de desempeñar la función semántica Manera.

c) La yuxtaposición, entendida como ausencia de coordinación (sea mediante conjunción sea mediante $\phi$ ), entre dos o más términos que están en el mismo nivel de la jerarquia sintáctica indica que tales términos desempeñan funciones semánticas distintas. Los dos criterios anteriores a) y b) permiten caracterizar positivamente la función semántica de un término. Pero la yuxtaposición sólo permite hacer una caracterización puramente negativa. La aplicación de este criterio se basa en el principio siguiente, complementario al expuesto en b): dos términos yuxtapuestos en el mismo nivel de la jerarquía sintáctica desempeñan funciones semánticas diferentes.

La aplicación de estos criterios no implica que considere los tradi-

G.; Monteil 1963; Ruijgh 1971; Rijksbaron 1976; y de la Villa 1986. Los ejemplos que se citan de otras obras y autores proceden de una revisión asistemática de los mismos. 
cionales como desprovistos de validez. En realidad, la identidad de desinencia gramatical (es decir, el criterio basado en la forma) es el más importante y el que puede ser aplicado de un modo más general. Igualmente, el significado del propio lexema con el que se combina el morfema o desinencia gramatical merece ser tomado en consideración, al menos como procedimiento tentativo (cf. Vester 1983: p. 47 ss.; de la Villa 1988). Sin embargo, los criterios que aquí vamos a aplicar, aparte de implicar el uso de éstos, presentan ciertos avances de detalle. Además, permiten poner en relación elementos gramaticales distintos que, tradicionalmente, se consideran de un modo atomizado.

3. Los criterios enumerados permiten establecer que al menos las siguientes formas y términos son susceptibles de expresar la función Manera en griego.

3.1. Un gran número de adverbios en $-\omega \varsigma,-\eta,-a,-o v,-a$. Los ejemplos de (2) muestran que los adverbios en - $\omega \varsigma$ que responden a interrogaciones parciales introducidas por $\pi \tilde{\omega} \varsigma$ expresan la función semántica Manera 2 :

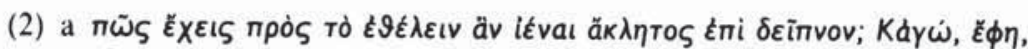

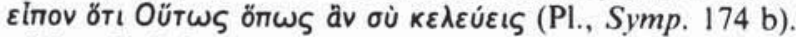

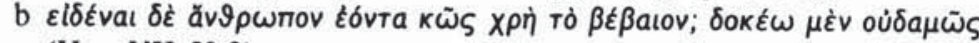
(Her. VII 50,2).

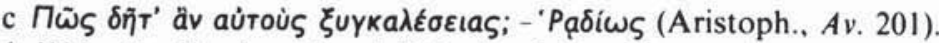

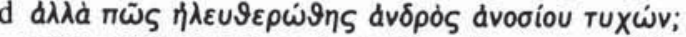

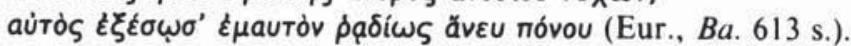

También otros adverbios con otras marcas formales o sin morfema gramatical pueden expresar la misma función Manera, como muestran los ejemplos de (3):

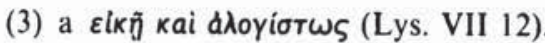

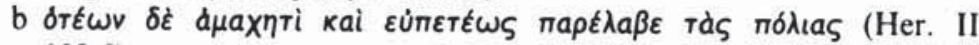
102,5).

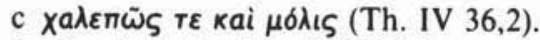

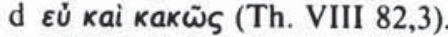

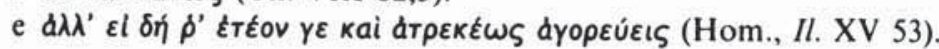

Conviene señalar que algunos adverbios que tienen idénticas marcas

${ }^{2}$ Existen algunos ejemplos en que a una pregunta introducida por $\pi \tilde{\omega} \varsigma$ se responde con un elemento que expresa Causa: cf. Th., V 92-3; Aristoph., Pl. 575 s.; Nub. 1434 s. Una discusión de los mismos se halla en Rijksbaron 1976: p. 22 ss. 
morfológicas a algunos de los recogidos en (2) y (3) parecen expresar funciones distintas de la de Manera. La terminación gramatical no es, pues, indicio inequívoco de que un determinado lexema desempeña una determinada función semántica. Algunos ejemplos de este tipo son los de (4):

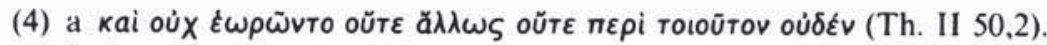

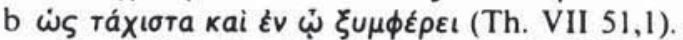

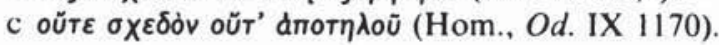

Para (4c) cf. (5), ejemplos que parecen indicar que los adverbios en - $\delta$ ov, excepto con el lexema $\sigma \chi \varepsilon^{-}$, expresan la función Manera:

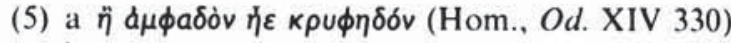

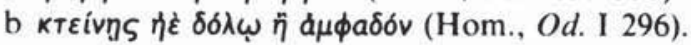

Los ejemplos precedentes muestran, por un lado, que la forma no es un procedimiento inequivoco para determinar la función expresada por un término, y, por otro, que el significado del lexema permite, al menos de modo tentativo, inferir qué función expresa un término ${ }^{3}$.

3.2. También el Dativo en un grupo numeroso de sustantivos es susceptible de expresar la función semántica Manera, como muestran los ejemplos de (6):

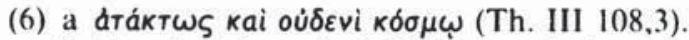

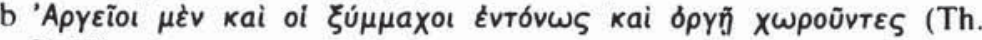
V 70).

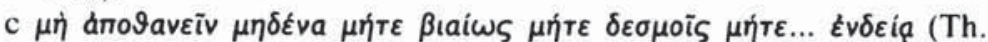
VII 82,2 ).

Junto a los ejemplos anteriores de coordinación entre un Dativo y una forma que expresa la función Manera, existen otros muchos ejemplos de yuxtaposición de un Dativo y otros elementos que expresan la función Manera. Así sucede en los ejemplos de (7):

${ }^{3}$ Ciertos ejemplos en que dos adverbios en - $\omega \varsigma$ están yuxtapuestos pueden ser explicados si se considera que uno se refiere a la manera en que se realiza el predicado verbal y otro a la manera en que se realiza la enunciación verbal. Algunos ejem-

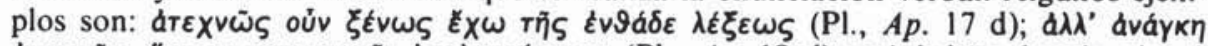

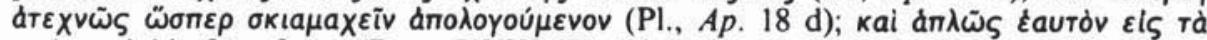

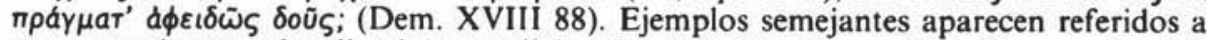
otras oraciones subordinadas: condicionales (cf. Pl., Symp. 220 d); causales (cf. Rijksbaron 1976); finales, en el tipo de "para que lo sepas, ayer no salió», donde la subordinada no se refiere a la finalidad de salió, sino a la de la enunciación. 


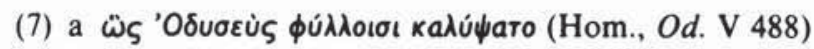

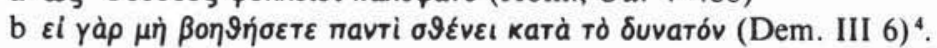

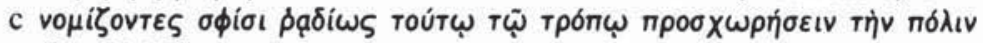
(Th. II 2,4).

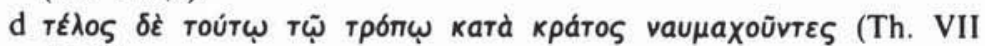
$41,1)^{4}$.

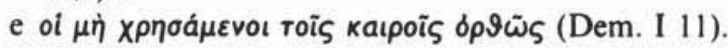

Los ejemplos de (7) muestran que el Dativo puede expresar otras funciones distintas de la de Manera (Instrumento, Beneficiario, etc.; cf. de la Villa 1988). Ello explica que el Dativo aparezca en unos contextos coordinado con adverbios en - $\omega \varsigma$, mientras que en otros está yuxtapuesto a términos que expresan Manera. Cuando el Dativo no aparece ni coordinado ni yuxtapuesto con otros elementos que permiten precisar la función del Dativo, el único criterio para determinar la función expresada por el Dativo es el propio lexema con el que se combina, así como el significado del lexema regente. Esto es lo que sucede en contextos como (8):

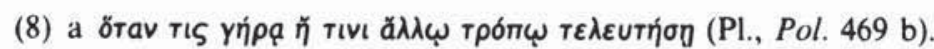

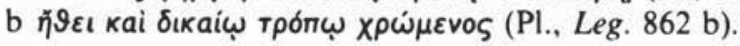

Para decidir si los Dativos de (8) expresan Instrumento o Manera, los únicos criterios proceden de los lexemas en Dativo y del lexema del predicado verbal.

También el Acusativo aparece coordinado con un término que expresa la función Manera en los siguientes ejemplos, al menos:

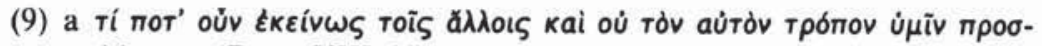

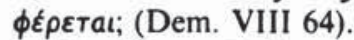

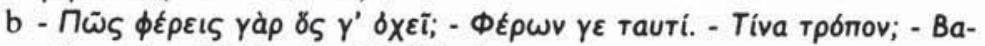
$\rho \varepsilon \omega_{\zeta}$ mávv (Aristoph., $R a .25 \mathrm{~s}$.).

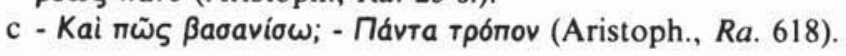

Como en todos los ejemplos el lexema es el mismo, cabe suponer que el lexema condiciona fuertemente la función.

3.3. Un buen número de sintagmas preposicionales expresa la función semántica Manera, al menos con determinados lexemas. Para los siguientes sintagmas preposicionales hemos hallado ejemplos que satisfacen alguno de los criterios que utilizamos: кaтá + Acusativo (10), żmí

\footnotetext{
${ }^{4}$ Para kará + Acusativo, cf. infra, 3.3, y Jiménez (1987).
} 
+ Dativo (11), $\boldsymbol{\varepsilon} \pi i$ + Genitivo (12), $\boldsymbol{\varepsilon} v+$ Dativo (13), $\dot{u} \pi \delta$ + Genitivo (14), $\boldsymbol{\varepsilon} \boldsymbol{k}+$ Genitivo (15). Es verosímil suponer que la consideración de un número más amplio de datos añadiría otros sintagmas preposicionales. Hay que señalar también que la función Manera en los sintagmas preposicionales suele estar asociada a determinados lexemas. Algunos ejemplos son:

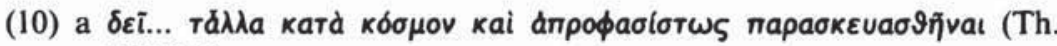
VI 72,5)

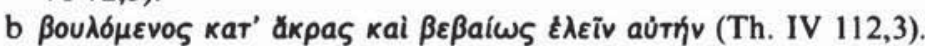

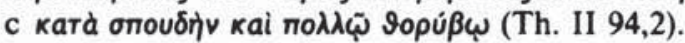

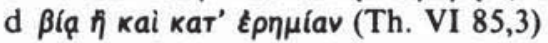

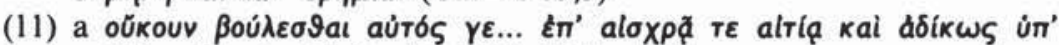

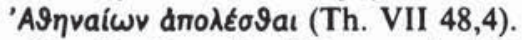

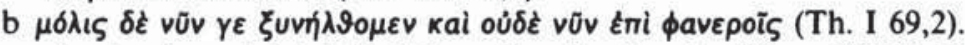

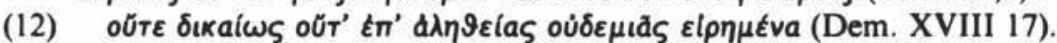

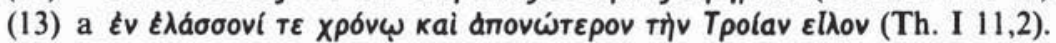

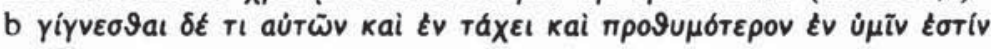
(Th. VI 92,1).

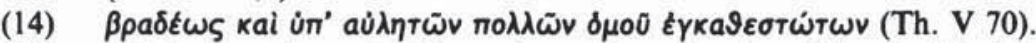

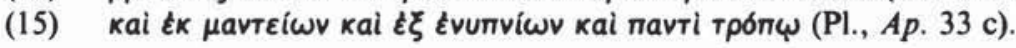

Estos sintagmas preposicionales, aparte de la función Manera, expresan otras funciones semánticas. Ello explica que aparezcan habitualmente yuxtapuestos con otros morfemas que desempeñan la función Manera (cf., por ejemplo, Th. IV 112,3).

3.4. Los sintagmas $u$ oraciones subordinadas introducidos por $\dot{\omega} \varsigma$ $\mathrm{y}$, en general, las demás conjunciones comparativas y consecutivas son susceptibles de desempeñar, al menos, la función semántica Manera, según muestran los ejemplos de (16):

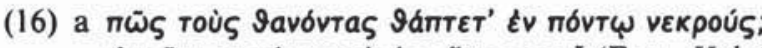

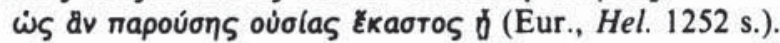

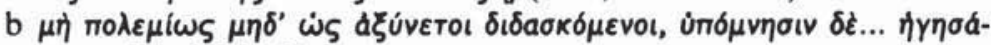
$\mu \varepsilon v o$ (Th. IV 17,3).

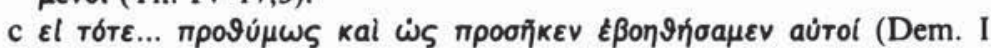
9).

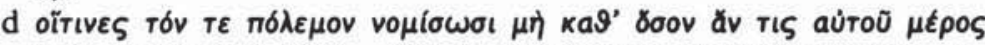

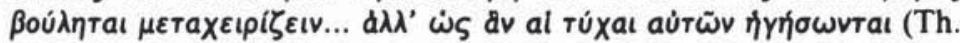
IV 18,4).

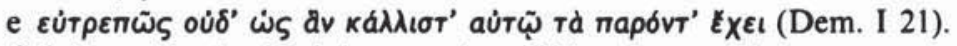

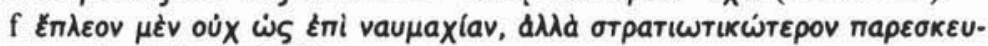
aquḱvoi (Th. II 83,3).

Por otro lado, los ejemplos de la épica que admiten dos análisis diferen- 
tes para $T \varepsilon$ muestran en todo caso que, aun cuando $T \varepsilon$ deba ser entendido como $\tau \varepsilon$ «épico", el origen de este uso remonta a una época en la que este tipo de coordinación era frecuente como tal (cf. Ruijgh 1971: p. 567 ss.). Un ejemplo de este tipo es (17):

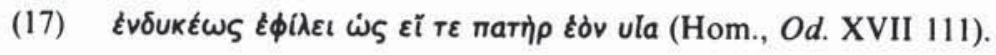

No he hallado ningún ejemplo de coordinación de subordinadas consecutivas con otras formas que desempeñan la función Manera. Sin embargo, los correlativos que expresan la función Manera son sumamente frecuentes con las consecutivas (cf. Monteil 1963: p. 330 ss).

3.5. También los participios absolutos pueden desempeñar, entre otras (cf. Crespo 1987), la función semántica Manera, como muestran los ejemplos de (18):

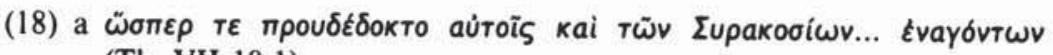
(Th. VII 18,1)

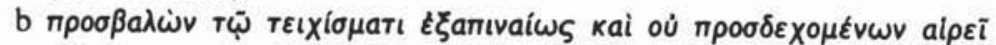
(Th. III 34,3).

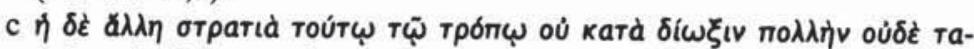

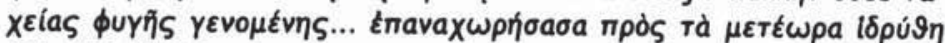
(Th. IV 44,2).

Cuando los términos coordinados son susceptibles de cumplir otras funciones semánticas aparte de la de Manera, como sucede en (18 c) (cf. supra, 3.3, a propósito de кaтá + Acusativo), existe la posibilidad de que ambos términos desempeñen otra función distinta (cf. 3.9).

3.6. También los participios concertados aparecen con frecuencia coordinados con términos que expresan la función Manera o en respuesta a preguntas parciales introducidas por el interrogativo $\pi \tilde{\omega} \varsigma$. Algunos ejemplos se recogen en (19):

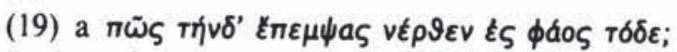

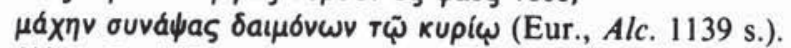

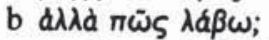

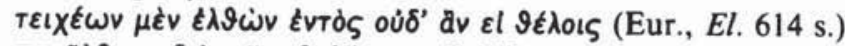

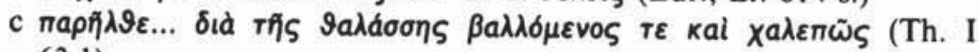
$63,1)$.

d каi $\pi \varepsilon \zeta \tilde{~ \kappa a i ~ v a u \mu a \chi о u ̃ v т \varepsilon \varsigma ~(D e m . ~ I I I ~ 24) . ~}$

Por supuesto, los participios concertados aparecen también coordinados 
con términos que expresan otras funciones distintas, como veremos infra (cf. 5.4).

3.7. También muchos adjetivos, sea cual sea su desinencia de caso, aparecen coordinados con procedimientos gramaticales que expresan la función semántica Manera. Algunos ejemplos son los de (20):

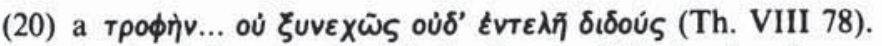

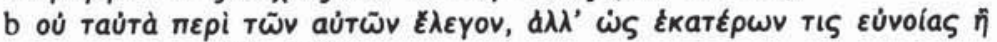
$\mu v \eta \dot{\mu \eta} \varsigma$ हैхо (Th. I 22).

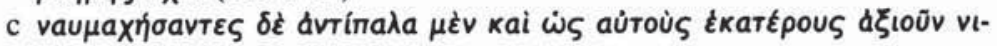
$\kappa a ̃ v(T h$. VII 34,6).

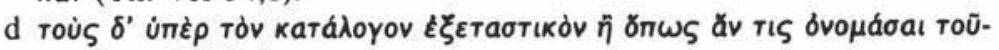
To (Dem. XIII 4).

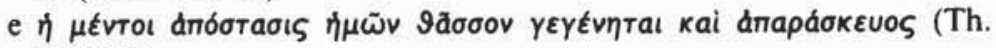
III 13,2).

Como veremos (cf. infra, 5.1), los adjetivos, en general, aparecen coordinados también con otros términos que expresan funciones semánticas distintas de la de Manera.

3.8. En conclusión, los criterios utilizados permiten establecer que los siguientes procedimientos pueden expresar Manera:

(21) a adverbios en - $\omega \varsigma, \eta,-a,-o v,-a,-\delta o v$ y otros,

b Dativo de muchos sustantivos, asi como el Acusativo rрб́rov, al menos,

c diferentes sintagmas preposicionales,

$\mathrm{d}$ oraciones y sintagmas introducidas por $\dot{\omega} \varsigma, \tilde{\omega} \sigma \pi \varepsilon \rho, \delta \pi \omega \varsigma, \tilde{\omega} \sigma \tau \varepsilon$, etc.,

e participios en construcción absoluta,

f participios en construcción concertada,

$\mathrm{g}$ adjetivos.

3.9. Como ya se ha indicado supra, la mayoría de los procedimientos enumerados en (21) son susceptibles de expresar no sólo la función Manera, sino también otra u otras funciones semánticas. Como consecuencia de ello, existen muchos ejemplos que, aun cuando satisfacen alguno de los criterios utilizados para determinar las funciones semánticas expresadas por los términos (cf. § 2), no permiten determinar con seguridad que es la función Manera la expresada por ambos términos. Así sucede en los ejemplos de (22):

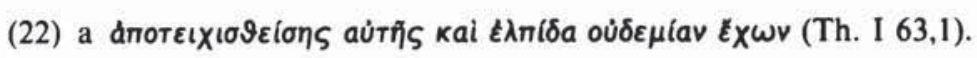




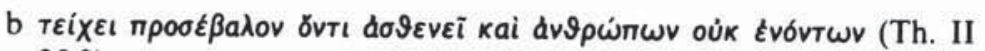
25,2).

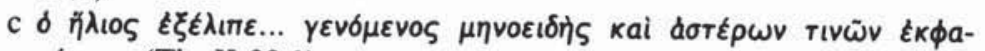
vévtwv (Th. II 28,1).

Tanto el participio absoluto como el participio concertado, elementos que aparecen coordinados en (22), pueden aparecer coordinados con elementos que expresan otras funciones además de la de Manera. De ahí que sólo la consideración de los lexemas coordinados en (22) permite inferir cuál es la función de ambos términos, que puede ser tanto Manera como otras.

Lo mismo sucede en los ejemplos de (23):

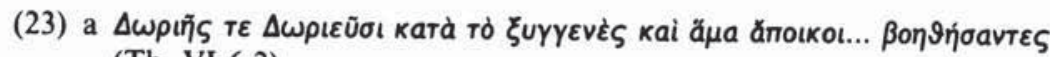
(Th. VI 6,2).

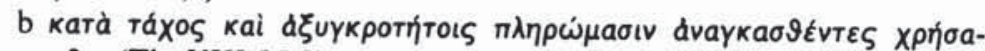
o૭ai (Th. VIII 95,2).

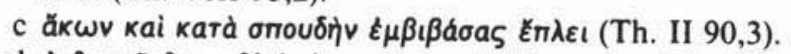

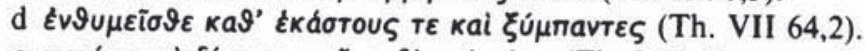

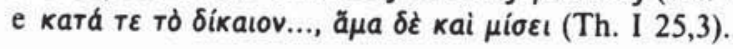

Los elementos coordinados en los ejemplos de (23), el sintagma кaтá + Acusativo y un adjetivo o un Dativo en (23 e), son susceptibles de expresar otras funciones aparte de la de Manera.

Lo mismo sucede cuando aparecen coordinados un adjetivo o un Dativo y un participio absoluto, como en (24).

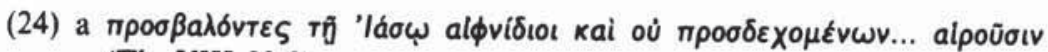
(Th. VIII 28,2).

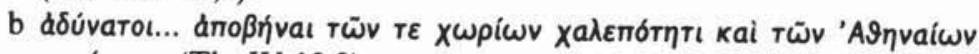
$\mu \varepsilon v \delta ́ v \tau \omega v$ (Th. IV 12,2).

La función semántica expresada por los términos coordinados sólo puede ser inferida a partir de la consideración de los lexemas (cf. otros ejemplos en 5.4).

Por otro lado, existen numerosos contextos en los que es dificil determinar con seguridad cuáles son los términos coordinados. Un ejemplo es (25):

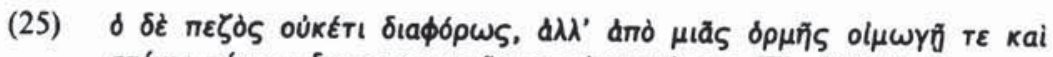

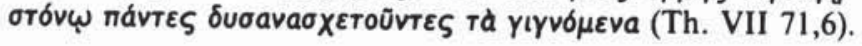

El adverbio en - $\omega \varsigma$ aparece coordinado bien con el sintagma preposi- 
cional que sigue, bien con návтєৎ, sin que sea posible determinar con seguridad cuál es el término coordinado con el adverbio.

La abundancia de estos tipos de ejemplos, en los que no es posible determinar con seguridad la función semántica expresada por dos o más términos (bien porque aparecen coordinados dos elementos que pueden expresar más de una sola función semántica, bien porque no existen indicios seguros para determinar la función aparte del propio lexema [cf. 3.1 y 3.2], bien porque no se puede establecer con seguridad cuáles son los términos coordinados) hace difícil abordar ciertos temas que la gramática debería describir. En efecto, sería deseable que la gramática estableciera reglas que pudieran dar cuenta de lo siguiente:

a) cuándo las formas morfológicas que pueden expresar más de una función semántica deben ser interpretadas como cumpliendo una $u$ otra función; por ejemplo, cuándo un Dativo expresa Manera y cuándo Instrumento u otra función que el Dativo es capaz de expresar;

b) qué lexemas son aptos para expresar cada función determinada; por ejemplo, qué condiciones debe reunir el lexema de un determinado sintagma preposicional como кaт́́ + Acusativo para que sea posible afirmar que expresa la función Manera y no, por ejemplo, la función Ubicación;

c) con qué tipos de predicados y, en general, elementos regentes es compatible o incompatible la aparición de un término que exprese, por ejemplo, la función Manera.

Naturalmente, para hallar una respuesta para estas cuestiones (tratadas por Vester 1983 para el latín), sería necesario tomar en consideración la totalidad de los ejemplos en los que uno u otro procedimiento expresa la función Manera. Ello exigiría reunir en un mismo conjunto todos los ejemplos que aparentemente expresan la función Manera, tanto aquellos que satisfacen alguno de los criterios que utilizamos para establecer la función semántica expresada por los términos (cf. § 2), como aquellos en los que sólo el lexema permite inferir de modo tentativo que expresan la función Manera. El resultado de este proceder sería la utilización indiscriminada de ejemplos de valor distinto para describir las reglas que den cuenta de las cuestiones planteadas en a)-c).

Por ello, dejando de lado las cuestiones planteadas en a)-c), nuestro propósito es ahora simplemente determinar las condiciones que regulan la distribución de los procedimientos enumerados en (21) para expresar la función semántica Manera.

4. Para explicar las condiciones que rigen la distribución de las 
formas que son susceptibles de expresar la función Manera, existen en principio varias posibilidades:

a) Puede suceder, en primer lugar, que existan diferencias de significado entre todas o algunas de las formas que expresan una misma función semántica. Así sucede, por ejemplo, en otras funciones, como

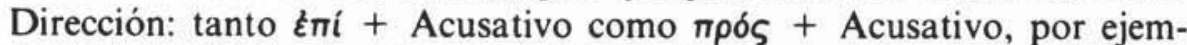
plo, desempeñan una misma función semántica, como prueban ejemplos como (26):

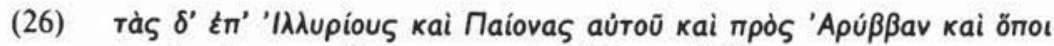

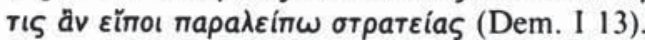

Sin embargo, el significado de ambos sintagmas preposicionales es diferente, porque estas preposiciones se combinan con lexemas que en cierta medida no son intercambiables.

b) En segundo lugar, existe la posibilidad de que las diferentes formas de expresar Manera, o algunas de ellas al menos, sean puras variantes libres. Esto es lo que parece suceder, por ejemplo, entre $\varepsilon^{\prime} v / \varepsilon v i$, прб́ $/ \pi \rho о т і$, etc., en la lengua homérica, independientemente de la interpretación diacrónica o dialectal que explique la presencia de ambas.

c) En tercer lugar, existe también la posibilidad de que la distribución esté regulada por una regla puramente mecánica, al menos en algunas de las formas susceptibles de expresar la función Manera. Así sucede, por ejemplo, en la distribución entre participio concertado y participio absoluto (cf. K.-G., II, p. 78).

d) En cuarto lugar, existe también la posibilidad de que algunos o todos los procedimientos de expresar la función Manera presenten ciertas restricciones de distribución que deban ser explicadas por otras causas independientes de las semánticas y mecánicas.

En todo caso, los ejemplos de $\S 3$ muestran que en griego hay que contar con una sola función Manera, y no con varias funciones distintas (Circunstancia, Motivo, Resultado, etc.), frente a lo que, según Vester (1983), ocurre en latín. Ningún dato en griego hace necesario proponer la existencia de estas funciones.

La distribución de los procedimientos enumerados en (21) para expresar la función Manera depende en parte del tipo de lexema con el que cada morfema es capaz de combinarse. Esto se puede representar mediante (27): 
(27)

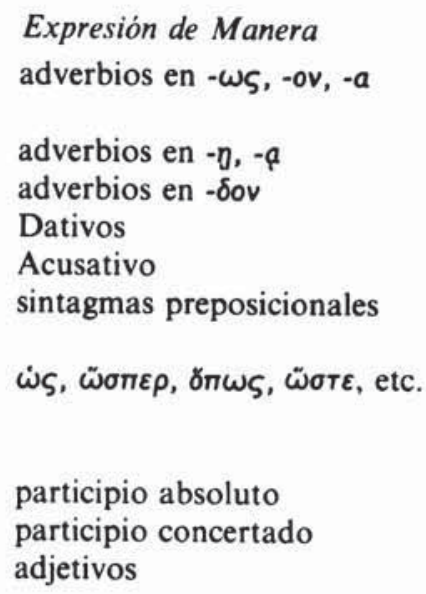

\author{
Tipo de lexema \\ adjetivos, pronombres no perso- \\ nales \\ sustantivos \\ verbos \\ sustantivos \\ тро́тог \\ sustantivos, pronombres perso- \\ nales \\ adjetivos, sustantivos, adver- \\ bios, verbos, pronombres, or. \\ subordinadas \\ verbos $\mathrm{y}$ oraciones subordinadas \\ verbos $\mathrm{y}$ oraciones subordinadas \\ adjetivos
}

Existen, pues, ciertas restricciones en las posibilidades de combinación, tanto en los diferentes tipos de lexemas como en los procedimientos para expresar la función Manera. Sólo las oraciones subordinadas o sintagmas introducidos por $\dot{\omega} \varsigma$, $\ddot{\sigma} \tau \varepsilon \rho, \delta \pi \omega \varsigma, \ddot{\omega} \sigma \tau \varepsilon$, etc., admiten ser combinados con cualquier tipo de lexema.

5. Según (27), la mayoría de los tipos de lexema puede combinarse con más de un procedimiento para expresar la Manera. Nuestro propósito es ahora determinar las condiciones que regulan la distribución de los procedimientos para expresar la Manera dentro de cada tipo de lexemas. En primer lugar, examinaremos las condiciones que regulan la distribución de las distintas formas de expresar Manera en los adjetivos (5.1); luego, en los adverbios (5.2), en los sustantivos y pronombres (5.3), y en los verbos y oraciones subordinadas (5.4). Finalmente, en 5.5 resumiré las conclusiones obtenidas.

5.1. La distribución de los tres procedimientos que, según (27), sirven para expresar la función Manera con los adjetivos está regulada por las siguientes condiciones. Los adverbios derivados en - $\omega \varsigma,-o v,-a$ expresan gramaticalmente la función semántica Manera, pero no indican formalmente a qué elemento están subordinados. El simple adjetivo, atributo o predicativo, expresa mediante la concordancia de la desinencia a qué elemento nominal está subordinado el adjetivo, pero no indica la función semántica de modo gramatical. Finalmente, el tipo de (28) expresa tanto la función semántica (a través de $\dot{\omega} \varsigma$ ), como el nivel de inserción en la jerarquía sintáctica de la frase (a través de la concordancia):

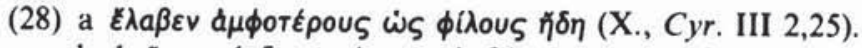

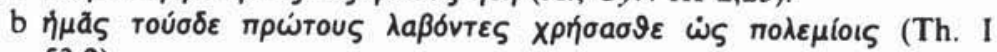
$53,2)$. 
Con respecto a los adverbios en $-\omega \varsigma,-o v,-a$ y a las construcciones del tipo (28), no parece necesario justificar detalladamente las afirmaciones precedentes. Baste simplemente señalar que los adverbios en - $\omega \varsigma$, -ov, - a no siempre dependen del predicado verbal de la frase. Sin embargo, es preciso justificar brevemente la afirmación de que los adjetivos no expresan ninguna función semántica mediante el lexema. Para ello hay que presentar ejemplos que muestren que los adjetivos son también susceptibles de estar coordinados con términos que expresen funciones semánticas distintas de la de Manera.

Algunos adjetivos aparecen coordinados con términos que expresan la función Tiempo en los ejemplos de (29):

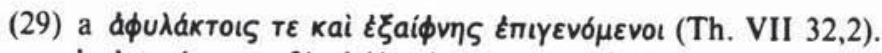

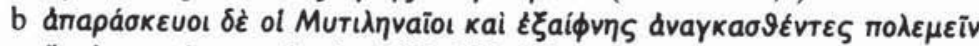
Eknhouv $\mu \varepsilon_{v}$ riva $\varepsilon$ moiouv (Th. III 4,3).

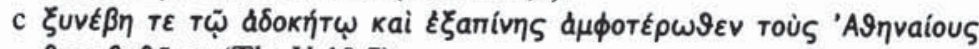

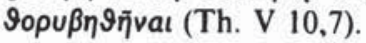

También algunos adjetivos se coordinan con términos que expresan la función Ubicación, como en los ejemplos de (30):

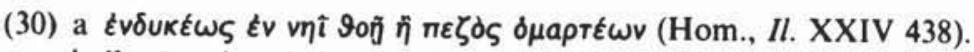

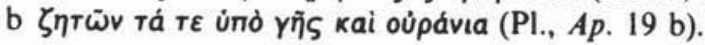

También hay adjetivos que aparecen coordinados con términos que expresan la función Origen, como en (31):

(31) oúd' трб́по (Th. I 76,2).

Igualmente, algunos adjetivos aparecen coordinados con términos que expresan la función Dirección, como en los ejemplos de (32):

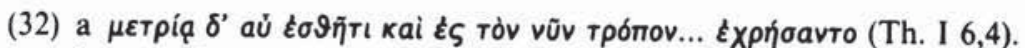

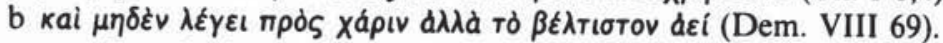

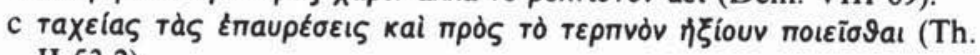
II 53,2 ).

Algunos adjetivos aparecen también coordinados con términos que expresan la función Extensión, como en (33):

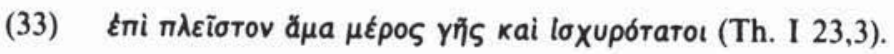


Algunos adjetivos también aparecen coordinados con otros sintagmas preposicionales o adverbios: $\mu \varepsilon т a ́$ + Genitivo (Th. II 15,2; III

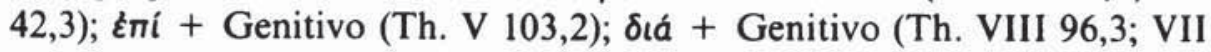
40,3; VI 34,2; VIII 43,4; VII 48,5); bாí $\sigma \omega$ (Hom., Il. III 218).

Los ejemplos precedentes, en los que distintos adjetivos aparecen coordinados con términos que cumplen diferentes funciones semánticas, admiten dos interpretaciones en principio: a) los adjetivos, cuya terminación expresa sólo la concordancia y no la función semántica, no desempeñan ninguna función mediante la que su lexema se relaciona con el término regente; b) los adjetivos, gracias al lexema, cumplen una función semántica con respecto al término regente (cf. Risselada 1984). Si la segunda interpretación es correcta, cada adjetivo será capaz de aparecer coordinado únicamente con términos que desempeñan una sola función semántica, la que corresponde a cada lexema. Por el contrario, si la primera interpretación es correcta y, en consecuencia, el adjetivo no se relaciona con el término regente por la función semántica que cumple en relación con él, sino por la relación sintáctica que marca la concordancia (cf. Ostrowski 1982), hay que esperar que cada adjetivo pueda aparecer coordinado con términos que desempeñan funciones semánticas diferentes entre sí.

La primera interpretación es la adecuada, porque existen ejemplos en los que un mismo adjetivo aparece coordinado con términos que desempeñan funciones semánticas distintas entre sí. Algunos ejemplos de este tipo son (34) y (35):

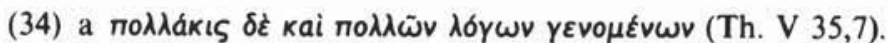

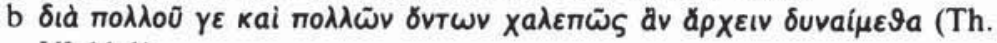
VI 11,1).

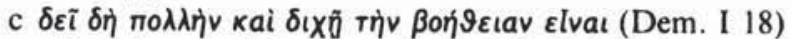

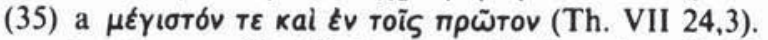

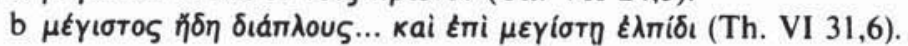

En los ejemplos (34) y (35) el mismo adjetivo aparece coordinado con términos que desempeñan funciones semánticas distintas entre sí. Estos datos indican que los adjetivos no expresan ninguna función semántica mediante su lexema, sino que se relacionan con el término regente mediante un procedimiento puramente sintáctico, no semántico, que está marcado por la concordancia.

La discusión precedente muestra que en griego existen, en términos generales, dos mecanismos de naturaleza distinta (semántica y sintáctica, respectivamente) para indicar las relaciones existentes dentro de la frase u oración. Por un lado, un término regido puede expresar (me- 
diante procedimientos gramaticales o mediante el propio lexema) la función semántica que desempeña con respecto a otro elemento, del que no se indica cuál es. Las desinencias casuales en los sustantivos (excepto en la aposición) marcan qué función desempeña el lexema con el que se combinan, pero no marcan con qué elemento se relacionan. Por el contrario, un término regido puede expresar con qué elemento se relaciona ( $\mathrm{y}$ ello se indica mediante la concordancia), pero no la función semántica que relaciona el lexema regido con el término regente. Existen, pues, dos sistemas en parte complementarios: el término regido expresa bien la función sin indicar respecto a qué elemento regente, bien la relación gramatical con el regente sin indicar la función. Este segundo sistema es característico de los adjetivos. En los ejemplos del tipo de (36):

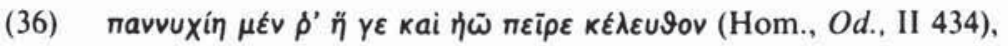

el adjetivo y el Acusativo coordinados marcan, respectivamente, la relación sintáctica con el término regente y la función semántica mediante la que el lexema regido se relaciona con el término regente.

Los resultados de la discusión precedente se pueden resumir así. La distribución de los procedimientos para expresar la función Manera en los adjetivos se rige por las siguientes condiciones: las marcas morfológicas adverbiales $-\omega \varsigma,-o v,-a$, etc., expresan la función del lexema con el que se combinan, pero no a qué elemento regente hay que atribuir tal función; los adjetivos en cuanto tales no expresan función, pero sí indican mediante la concordancia con qué elemento regente hay que relacionar su lexema; finalmente, $\dot{\omega} \varsigma$ + Adjetivo expresa tanto la función, como el elemento con el que el lexema se relaciona.

5.2. Como hemos visto (cf. 3.1), en los lexemas tradicionalmente clasificados como adverbios la función Manera puede ser indicada por: a) ciertas marcas gramaticales ( $-\omega \varsigma,-o v,-a$, etc.); b) el propio lexema $(\mu \delta \dot{\lambda} \iota \varsigma, \varepsilon \dot{v}$, etc.); c) además, la adición de una conjunción como $\dot{\omega} \varsigma$,

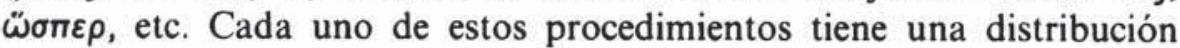
distinta: el uso de a) constituye un procedimiento morfológico productivo; el uso de b) depende de la existencia en el léxico de una forma específica; el uso de c), particularmente frecuente con los superlativos adverbiales en -ov y - $a$, marca la función, que, mediante la terminación, sólo está indicada por la ausencia de concordancia con otro elemento. Además, $\dot{\omega} \varsigma$ + adverbio en - $\omega \varsigma$ aparece sobre todo con ciertos lexemas adverbiales que indican tanto la Manera del enunciado como la Manera de la enunciación. Un ejemplo de este tipo es (37): 


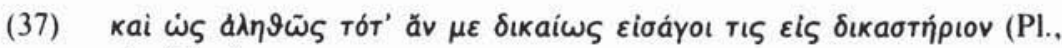
Ap. 29 a).

La presencia de $\dot{\omega} \varsigma$ puede ser explicada como un procedimiento formal para marcar el grado de inserción diferente de los dos adverbios que expresan la función Manera: el primero se refiere a la manera en que se hace la enunciación, mientras que el segundo expresa la manera del predicado enunciado. La construcción del tipo de $\dot{\omega} \varsigma$ ả $\lambda \vartheta \vartheta \tilde{\omega} \varsigma$ aparece particularmente con determinados lexemas adverbiales que aparentemente se documentan en yuxtaposición con otros elementos que expresan la función Manera. Ambas particularidades pueden ser explicadas si se parte de la observación de que estos lexemas adverbiales pueden referirse tanto a la manera del enunciado como a la manera de la enunciación ${ }^{5}$.

5.3. La función Manera puede ser expresada en los sustantivos y pronombres personales mediante varios procedimientos gramaticales: a) algunos adverbios en $-a,-\eta$; b) el Dativo de ciertos lexemas (y el Acusativo тро́поv, al menos); c) un cierto número de sintagmas preposicionales; d) $\dot{\omega} \varsigma$ + sustantivo o pronombre personal o reflexivo. La distribución de estos procedimientos, en general, está fuertemente condicionada por el lexema del sustantivo. Asi, el análisis de una forma en $-a,-\eta$

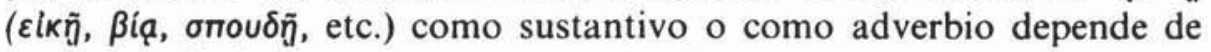
que en cada lexema sea o no productivo el paradigma morfológico completo. Igualmente, el Dativo expresando la función Manera parece excluir los lexemas de animados o de nombres de lugar. Igualmente, los pronombres personales y reflexivos sólo parecen documentarse en esta función con $\dot{\omega} \varsigma$ (casi siempre con Nominativo o Acusativo, cf. Ruijgh 1971: p. 579) o con катá + Acusativo. Igualmente, la elección de la preposición parece estar condicionada por el lexema, y con frecuencia es posible reconstruir una previa función local en el sintagma preposicional.

5.4. Entre los distintos procedimientos para expresar la función Manera con lexemas verbales y oraciones subordinadas, las diferencias son análogas a las establecidas en 5.1 para los adjetivos: el participio concertado no expresa ninguna función semántica, sino sólo la relación sintáctica marcada por la concordancia; $\dot{\omega} \varsigma$ + verbo expresa la función, pero no el nivel de inserción de la subordinada; $\dot{\omega} \varsigma$ + participio

\footnotetext{
${ }^{5}$ Cf. supra, nota 3.
} 
concertado expresa tanto la función como el nivel de inserción; las desinencias $-\delta o v,-\delta \eta v,-t$, $-\tau l$, etc., representan procedimientos lexicalizados y no productivos en griego clásico; finalmente, para el participio absoluto, cf. Crespo 1987.

Las condiciones expuestas para explicar la distribución de los procedimientos para expresar la Manera en estos contextos sólo requiere cierta justificación en el caso de los participios concertados. Trataremos, pues, de justificar ahora la afirmación de que los participios concertados no expresan función semántica.

Como hemos visto (3.6), un participio concertado puede aparecer coordinado con términos que expresan la función Manera y en respuesta a preguntas parciales sobre la manera. Además, el participio concertado aparece con frecuencia coordinado con términos que expresan la función Tiempo ubi, como en (38):

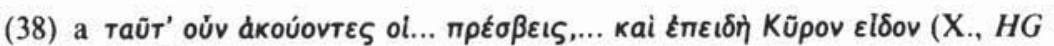
I 4,4).

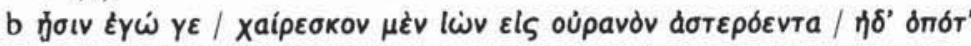

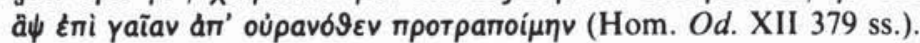

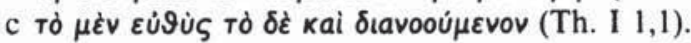

Igualmente, los participios concertados aparecen también como respuesta a elementos que expresan Causa, como en los ejemplos de (39):

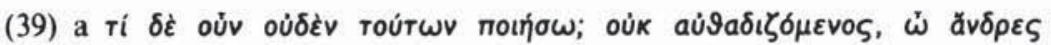

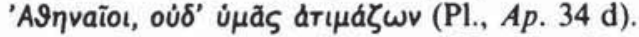

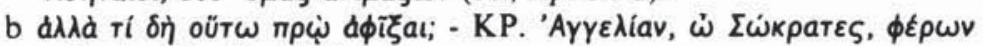

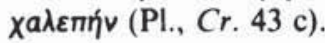

En otros contextos un participio concertado aparece coordinado con un Dativo que probablemente expresa también Causa. Sin embargo, el hecho de que el Dativo sea capaz de expresar otras funciones semánticas, además de Causa, hace la interpretación de la función expresada por el Dativo más insegura (cf. 3.2 y 3.9). Algunos ejemplos son los de (40):

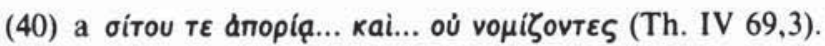

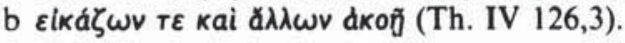

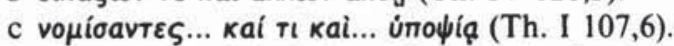

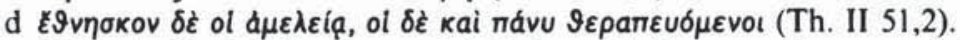

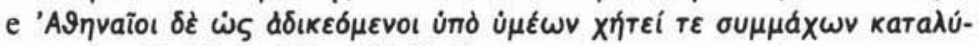

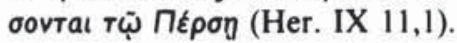


También hay ejemplos de participios concertados coordinados con elementos que expresan Finalidad. Algunos ejemplos son los de (41):

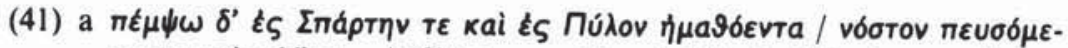

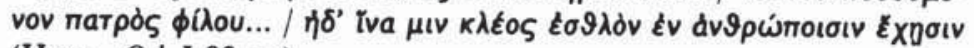
(Hom., Od. I 93 ss.).

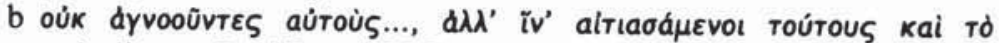

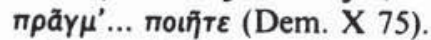

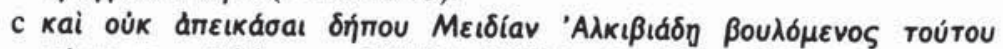

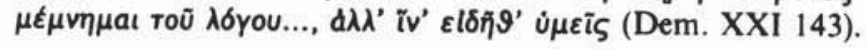

También los participios concertados aparecen coordinados con términos que expresan otras funciones, como en los ejemplos de (42):

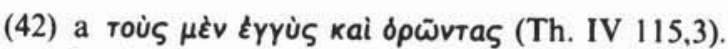

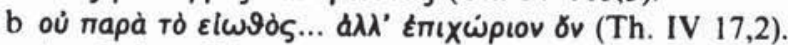

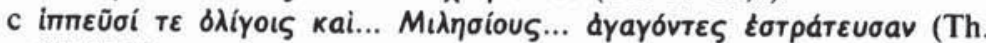
IV 53,1$)$.

No he hallado, sin embargo, ejemplos en los que un mismo participio concertado esté coordinado con términos que expresan funciones semánticas distintas. Aun así, los ejemplos precedentes permiten sostener que los participios concertados no expresan ninguna función semántica mediante su lexema, sino que están relacionados con el término regente por un procedimiento puramente sintáctico marcado por la concordancia gramatical (cf. 5.1).

En conclusión, con los lexemas verbales y oraciones subordinadas, los distintos procedimientos para expresar la función Manera se distribuyen conforme a condiciones precisas: el participio concertado no expresa la función, sino la pura relación gramatical marcada por la concordancia; las subordinadas comparativas o consecutivas, por el contrario, expresan gramaticalmente la función mediante la conjunción introductoria, pero no indican el nivel de inserción; además, existen ciertas desinencias que expresan la función Manera con lexemas verbales, que constituyen un procedimiento lexicalizado y no productivo.

5.5. Los resultados obtenidos de la discusión precedente sobre las diferentes marcas para expresar la función Manera dentro de cada tipo de lexemas (adjetivos, adverbios, sustantivos y pronombres personales, $\mathrm{y}$ verbos $\mathrm{u}$ oraciones subordinadas) se pueden resumir así.

Los procedimientos gramaticales que, según § 3, expresan Manera se combinan con los siguientes lexemas de acuerdo con la distribución de (43): 


\begin{tabular}{|c|c|c|c|c|}
\hline \multirow[t]{2}{*}{ lexemas } & \multicolumn{2}{|c|}{$\begin{array}{l}\text { relación funcional } \\
\text { (= función) }\end{array}$} & \multirow[t]{2}{*}{$\begin{array}{l}\text { relación sintáctica } \\
(=\text { concordancia })\end{array}$} & \multirow[t]{2}{*}{$\begin{array}{c}\text { relación funcional } \\
\text { y sintáctica }\end{array}$} \\
\hline & $\begin{array}{l}\text { por un } \\
\text { morfema }\end{array}$ & $\begin{array}{l}\text { por el } \\
\text { lexema }\end{array}$ & & \\
\hline adjetivo & $-\omega s$ & & adjetivo & $\dot{\omega} s+$ adjetivo \\
\hline adverbio & $\begin{array}{c}-\omega s \\
\dot{\omega}+-o v /-a \\
\dot{\omega}_{s}+a d v .\end{array}$ & $\begin{array}{l}\mu \delta \lambda \iota s, \\
\varepsilon \dot{v}, \text { etc. }\end{array}$ & & \\
\hline $\begin{array}{l}\text { sustantivo } \\
\text { y pronombre } \\
\text { personal }\end{array}$ & $\begin{array}{l}\text { Dativo } \\
\text { sintagma } \\
\text { prepos. }\end{array}$ & $-a,-n$ & & $\dot{\omega} \varsigma$ + sustantivo \\
\hline $\begin{array}{l}\text { verbo y } \\
\text { oración } \\
\text { subordinada }\end{array}$ & $\begin{array}{c}\dot{\omega} s+\text { verbo } \\
\text { Participio } \\
\text { absoluto }\end{array}$ & $\begin{array}{l}-\delta o v,-\delta \eta v \\
-1, \text { etc. }\end{array}$ & $\begin{array}{l}\text { participio } \\
\text { concertado }\end{array}$ & $\begin{array}{l}\dot{\omega} s+\text { participio } \\
\text { concertado }\end{array}$ \\
\hline
\end{tabular}

Los resultados expuestos en (43) muestran que los procedimientos utilizados en griego para expresar Manera constituyen un sistema relativamente homogéneo y organizado. Este sistema general resulta aún más coherente si se tienen en cuenta las dos observaciones siguientes.

En primer lugar, ciertos ejemplos de aposición entre sustantivos se corresponden funcionalmente con los adjetivos y participios concertados en la misma columna de elementos que expresan la relación sintáctica, no funcional, mediante la coincidencia de desinencia casual. Es decir, la aposición en los sustantivos equivale a los adjetivos y a los participios concertados en los lexemas nominales. Aun así, como era de esperar, no he hallado ejemplos en los que un sustantivo en aposición aparezca coordinado, o como respuesta, con un elemento que expresa la función semántica Manera. Conviene, sin embargo, señalar que existen muchos ejemplos de $\dot{\omega} \varsigma$ + sustantivo, equivalentes a la aposición de (44):

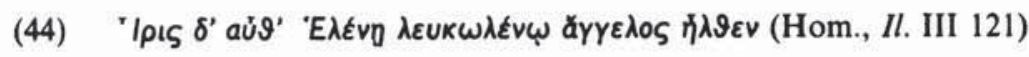

En segundo lugar, conviene observar con respecto a (43) que los procedimientos para expresar Manera en los adjetivos y adverbios presentan una distribución complementaria. Ello quiere decir que, al menos en la expresión de la función Manera, la distinción tradicional entre adjetivos y adverbios que expresan Manera no existe. Unos y otros lexemas aparecen en distribución complementaria (cf. Lyons 1968: p. 325 ss.).

En resumen, los procedimientos para expresar la función Manera se 
distribuyen de acuerdo con los tipos de lexemas con los que cada forma de expresar Manera es capaz de combinarse. Dentro de cada tipo de lexemas, la distribución de las formas susceptibles de expresar Manera está también regida por condiciones precisas. En la mayoría de los ejemplos la distribución depende de la elección entre expresar la relación funcional, la relación sintáctica marcada por la concordancia, o ambas.

6. Las conclusiones obtenidas mediante la aplicación de los criterios expuestos en $\S 2$ se pueden resumir así:

a) En griego existe una función semántica, que denominamos Manera, que indica gramaticalmente que el lexema subordinado se relaciona con un elemento regente expresando el modo en que éste o la enunciación se realizan. Esta función, distinta de Instrumento, Causa y otras, se expresa mediante un número limitado de procedimientos gramaticales y léxicos (cf. 3.8).

b) Cada tipo de lexemas y cada morfema gramatical que expresa la función Manera presenta ciertas restricciones de combinabilidad. Estas restricciones explican en parte la distribución de los procedimientos existentes para indicar la función Manera (cf. 4).

c) Incluso dentro de cada tipo de lexemas existen generalmente varios procedimientos para expresar la función Manera. La distribución en cada tipo de lexemas se explica casi siempre por la existencia de ciertas diferencias entre cada procedimiento de expresar Manera: unos procedimientos expresan gramatical o léxicamente la función; otros expresan la relación puramente sintáctica mediante la concordancia, y otros expresan ambas (cf. 5.5).

d) La relación entre los elementos de una oración se marca básicamente mediante dos mecanismos de naturaleza distinta: la expresión de la función semántica (mediante un morfema o el lexema) que el regido desempeña con respecto al regente; y la expresión de la relación puramente sintáctica, marcada por la concordancia. Este segundo tipo es propio de los adjetivos y participios concertados (cf. 5.1 y 5.4).

Emilio Crespo

\section{REFERENCIAS BIBLIOGRÁFICAS}

E. Crespo. 1987: "Participio absoluto y subordinada adverbial», Actas del VII Congreso Español de Estudios Clásicos, Madrid 1987 (en prensa).

S. C. Dik. 1968: Coordination. Its implications for the theory of general linguistics. Amsterdam: North Holland. 
S. C. Dik. 1980: Studies in Functional Grammar. Londres: Academic Press.

M. D. Jiménez. 1987: "Kará-Ac.: funciones semánticas y significado", Actas del VII Congreso Español de Estudios Clásicos, Madrid 1987 (en prensa).

K.-G.: R. Kühner-B. Gerth: Ausführliche Grammatik der griechischen Sprache. Satzlehre, Hannover 1898-1904, Hahnsche Buchhandlung $(=1966$, Darmstadt: Wiss. Buchgesellschaft).

J. Lyons. 1968: Introduction to theoretical linguistics. Cambridge: Cambridge University Press.

P. Monteil. 1963: La phrase relative en grec ancien. Paris: Klincksieck.

M. Ostrowski. 1982: "Zum Konzept der Kongruenz", en Apprehension. Das sprachliche Erfassen von Gegenständen. Teil I: Bereich und Ordnung der Phänomene, ed. H. Seiler-C. Lehmann, pp. 252-269. Tübingen: Gunter Narr.

H. Pinkster. 1972: On Latin Adverbs. Amsterdam: North Holland.

A. Rijksbaron. 1976: Temporal and causal conjuntions in ancient Greek. Amsterdam: Hakkert.

A. Rijksbaron. 1984: The syntax and semantics of the verb in classical Greek. An Introduction. Amsterdam: Gießen.

R. Risselada. 1984: "Coordination and juxtaposition of adjectives in the Latin NP", Glotta 62, pp. 202-231.

C. J. Ruijgh. 1971: Autour de TE épique. Amsterdam: Hakkert.

E. Vester. 1983: Instrument and manner expressions in Latin. Assen: Van Gorcum.

J. de la Villa. 1986: Sintaxis de los adverbios en griego antiguo. Análisis funcional. Madrid (tesis doctoral).

J. de la Villa. 1987: "Las funciones de los elementos nominales: criterios para su identificación en griego y latin", $C F C$ (en prensa).

J. de la Villa. 1988: "Caractérisation fonctionelle du dativ grec», Glotta (en prensa). 